\title{
Mathematical simulation of motion of working medium at finishing-grinding treatment in the oscillating reservoir
}

\author{
A. G. Mamalis • A. I. Grabchenko • A. V. Mitsyk • \\ V. A. Fedorovich • J. Kundrak
}

Received: 24 April 2013 / Accepted: 12 August 2013 / Published online: 5 September 2013

(C) Springer-Verlag London 2013

\begin{abstract}
The results of mathematical simulation have been carried out for the pattern of working medium motion providing the technological process of finishing-grinding treatment in an oscillating reservoir. With use of physics laws, it is ascertained and grounded that the flow of granules at the plane wall of reservoir is travelling oppositely to the source of vibrations, whereas the granules are drifting on the cycloidtrochoid trajectories from the wall of reservoir, where the looped displacement is maximal, to the center of reservoir in which the shift of granules is reduced to minimum because of damping and dissipation effect. The received theoretical regulations have a fundamental nature and can be used at the account of technological parameters of designed vibration machines.
\end{abstract}

Keywords Grinding · Finishing · Vibrations · Mathematical simulation

\section{Introduction into the finishing-grinding vibration treatment in the oscillating reservoir}

At the vibration treatment, the parts of different sizes, masses, and shape of surface, and also granular working medium, are

\footnotetext{
A. G. Mamalis $(\square)$

Project Center for Nanotechnology and Advanced Engineering (PC-NAE), NCSR "Demokritos", Athens, Greece

e-mail: mamalis@ims.demokritos.gr
}

\author{
A. I. Grabchenko · A. V. Mitsyk • V. A. Fedorovich \\ Department of Material Cutting and Cutting Tools, National \\ Technical University "Kharkiv Polytechnic Institute", \\ Kharkov, Ukraine \\ J. Kundrak \\ Department of Production Engineering, University of Miskolc, \\ Miskolc, Hungary
}

loaded into the vibration machine reservoir mounted on the elastic links. The forced oscillations are revealed to the reservoir. Then, from the working reservoir's surfaces such as their walls and bottom, the vibrations are passed into the depth of the working medium layer by layer that provides oscillating motion and intensive intermixing of all of the loaded mass. The vibration processing is being conducted in the circulating flows of reservoir's loading by way of micro-cutting and elastic-plastic deforming. As a result, the imperfect layer of metal is removed and the roughness of surface of the parts diminishes.

The vibration machines with «U»-shaped reservoir, the elastic links of which are presented by the cylindrical springs of different inflexibility, are mostly used. Such technological system creates bicomponent (binary) oscillation, the principal scheme of which consists of that load with mass $m$, connected eccentrically in radial direction with a shaft in the distance $r$ from its axis, and which accomplishes rotational motion with angular velocity $\omega$ (Fig. 1). Forcing force $F$, which is the result of forces $F_{y}$ and $F_{x}$ created by inertia vibration exciter in vertical and horizontal directions accordingly, is determined by the dependence $F=m r \omega^{2}$. As the reservoir of vibration machine leans upon the cylindrical springs, force $F$ causes the vibrations of reservoir in relation to an axis, passing through its center of gravity.

The reservoir of vibration machine accomplishes harmonic vibrations on two axes $O X$ and $O Y$. Thus, the beginning of the plane system of co-ordinates is on the axis of the shaft of vibration exciter in its static state. We will analyze the pattern of vibrations of reservoir in relation to the axis $O Y$. In a vertical plane, only vertical force $F_{y}$ renders action on a reservoir. The force $F_{y}$ submits to a sinusoidal law and is determined by the dependence $F_{y}=m r \omega^{2} \sin \omega t$.

Total mass $\sum M$ of mobile part of vibration machine, presented by the masses of reservoir, working medium and processed parts, in static position causes the sinking of springs 


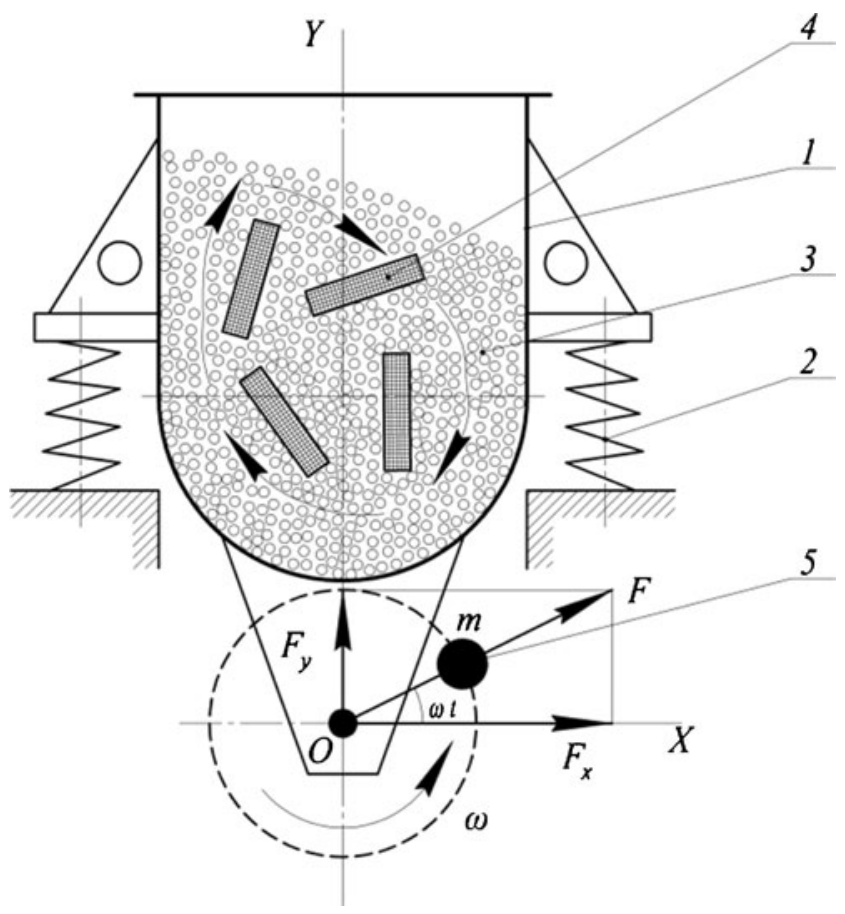

Fig. 1 Scheme of the technological system with bicomponent oscillation: 1 the processed parts, 2 a reservoir, 3 a working medium, 4 an elastic suspension, 5 vibration exciter

of elastic suspension $F_{y}$ on a value $\lambda_{y}$ which, taking into account their total inflexibility $\sum j_{y}$ in the direction of axis $O Y$, is equal $\lambda_{y}=\frac{\sum M}{\sum j_{y}}$.

In the process of operation of vibration machine, the oscillations of its mobile part submit to the law of rectilinear alternating motion in relation to the axis $O Y$. Differential equation of such motion looks like $\sum M \frac{d^{2} y}{d t^{2}}+\beta \frac{d y}{d t}+\sum j_{y} \lambda_{y}=F \sin \omega t$, where $\sum M \frac{d^{2} y}{d t^{2}}$ is force, caused by the action of mass of mobile part; $\beta$ is a coefficient of damping of vibrations in mobile part; $\beta \frac{d y}{d t}$ is force of resistance, causing the fading of vibrations in mobile part; and $\sum j_{y} \lambda_{y}$ is evocative force of springs of the reservoir's elastic suspension.

For the stable reservoir's oscillating motion, which is related to sinking of springs of its elastic suspension, the general solution of differential equation of motion looks like $y=A_{y} \sin (\omega t+\varphi)$, where $\varphi$ is an angle of translation of phases between the direction of action of forcing force of vibration exciter and direction of motion of center of the reservoir's masses.

Thus, the value of amplitude $A$ of reservoir's vibrations is direct proportionally to the forcing force $F$ of inertia vibration exciter, which changes its value direct proportionally to the square of frequency $\omega^{2}$ of reservoir's vibrations. For the examined case, the value of vertical amplitude of reservoir's vibrations $A_{y}$ can be presented in a form: $A_{y}=N_{y} \frac{m r}{M}$, where $N_{y}$ is a coefficient of dynamic force.

Because the total inflexibilities of springs of elastic suspension of reservoir on the axes $O X$ and $O Y$ are not equal, the equality between vertical $A_{y}$ and horizontal $A_{x}$ amplitudes of reservoir's vibrations is absent, that is $A_{y} \neq A_{x}$. Consequently, the motion of reservoir in all of the cases will take a place on ellipsoidal or near to it trajectory, which is described by the equation $\frac{x^{2}}{A_{x}^{2}}+\frac{y^{2}}{A_{y}^{2}} \approx 1$.

As the sizes of semi-axes of ellipse of motion trajectory depend on the inflexibility of springs of elastic suspension in the direction of axes $O X$ and $O Y$, the trajectory of reservoir's motion can change on a shape from a vertical ellipse to the circumference. So the ellipsis coefficient $K_{A}$ of trajectory, which is equal to the relation of values of vertical $A_{y}$ and horizontal $A_{x}$ amplitudes of reservoir's oscillations, takes the values $K_{A}=\frac{A_{y}}{A_{x}}=(\cong 1,0) \ldots(\geq 2,5)$.

The reservoir's oscillations are accomplished on the closed trajectories, so every granule and layers of working medium and all of mass of loading, including the processed parts are in circulating motion. The velocity of circulating motion is considerably less than the angular speed $\omega$ of unbalances of the inertia vibration exciter.

\section{The physical approaches to the explanation of motion pattern of working medium}

The monitoring of motion pattern of free working medium during finishing-grinding vibration processing [1-3] allowed the setting of conformity to the law, according to which the working medium granules participate in two kinds of motion. The finishing-grinding vibration treatment was conducted in a reservoir, which performed plane oscillating motions, the source of which was an external inertia vibration exciter [4, 5] connected to the reservoir so that their axes were parallel [6].

The first of the exposed types of motion, associated with the oscillation of working medium, can be characterized as close to the chaotic movement of the granules in relation to each other. The second type of motion, related to the circulation of the working medium, characterizes the formation of multi-layered by-wall flow of granules, directed at different sections of the trajectory of motion of the reservoir, as both along its planed walls, dynamically affecting the working medium and perpendicular to it $[7,8]$. Thus total motion of the working medium granules during the period of vibrations of the reservoir is opposite to the motion of its planed walls, and the velocity of granules presents the vector sum of velocities of both types of motions [9]. It should be noted that under the by-wall surface flow is understood that the flow of granules, which directly contacts the workings of surfaces of the oscillating reservoir, dynamically affects the working medium loaded into the reservoir $[10,11]$.

During the modeling of motion of working medium in the process of vibration treatment, the hypothesis used is that a 
behavior of oscillating granules massif on its character and physical symptoms may be analogical to the behavior of gas molecules, which interact with each other according to the laws of collisions of spherical bodies, which are accomplishing the rectilinear motions between collisions without the account of action of gravity forces [12].

The size of molecules in the gas models is taken into account at the characterization of the phenomena of transfer, i.e., at the estimation of viscosity, heat conduction, and diffusion. In this case, the gas molecules are associated with the bodies of spherical shape. The size of molecules is taken into account most fully when modeling the behavior of the real gas that leads to more high-quality explanation of flowing of phase transitions.

It should be noted that the basic hypothesis of kinetic theory of gases consists of the physical properties of gases that are determined only by the motion of molecules and does not depend on their internal structure. Gas molecules are in permanent motion and do not interact with each other except of those cases when they are located in direct closeness in the distance equal to their averaging sizes. Such interaction is examined as elastic collision. So it is accepted in the theory of gases that a molecule presents itself an absolutely elastic sphere and forces arising up in the process of motion are caused by the collision [13]. The size of such sphere is named by the gas-kinetic diameter of molecule [14]. The motion of molecules and their collisions with each other has random chaotic pattern. Detailed study of these processes, based on the solution of Newton's equations is virtually impossible. So the kinetic theory of gases applies the statistical method based on the use of averaged values such as the concentration of molecules in the volume, their average velocities, pressure of molecules, etc. In addition, the function of distribution of molecules in the space velocity impulses is entered. Such approach allows us to derive the relationships to calculate the motion of the gas at the macro-level, which is to receive the Navier-Stokes equations. In this connection, the conformity of the properties of gases, calculated theoretically and produced experimentally, is the criterion of the correctness of the model.

In the process of finishing-grinding vibration treatment, flowing in the oscillating reservoir, granules of working medium, having a shape near to spherical, are in permanent circulating motion which is provided by the operation of inertia vibration exciter. Thus the granules interact between each other only in the moment of their collisions. It should be noted that the number of working medium's granules involved in the process of vibration processing much less than the number of gas molecules in the same volume. However, their number $\left(>10^{5}\right)$ is sufficient for the application of the central limit theorem and introduction of function of the distribution of the granules on the velocities and the coordinates [15].
Consequently, the basic postulates of the kinetic theory of gases are saved to a full degree to describe the physical conduct of granules of working medium, applied in finishing-grinding vibration treatment. In this connection, the use of methods of calculation within the framework of kinetic theory of gases is fully permissible for the mathematical simulation of kinematics of motion of working medium and also the processes of distribution of power impulses in it. The legitimacy of such approach is confirmed by the adequacy of design parameters of motion of working medium's granules to the parameters, being registered in the experiment.

Thus, we can assume that the basic hypotheses used in the theory of ideal and real gases are practically fully acceptable to the description of physical situations that characterize the behavior of the working medium granules, which under an influence of low-frequency vibrations are accomplishing the movements, necessary to achieve technological result of finishing-grinding treatment of parts in the reservoir of vibration machine. Consequently, the attempt to describe the motion of working medium granular flow by gas dynamics equations is under itself a good reason. Such an approach to the description of kinematics and dynamics of free working mediums has been developed in a number of studies [16], the results of which show perspective of the accepted approach for explanation of behavior of granular materials, being under the effect of powerful periodic actions.

\section{Initial equations and boundary conditions of motion of the working medium}

We will consider character of motion of elementary volume of granules of the working medium at a planed surface $B$, each point of which from multiple number $O, O_{1}, O_{2}, \ldots, O_{i}$ simultaneously accomplishes flat oscillating motions with an amplitude $\grave{A}$ and frequency $\omega$ in relation to the coordinate axes $O X$ and $O Y$ on the trajectory close to the circle (Fig. 2).

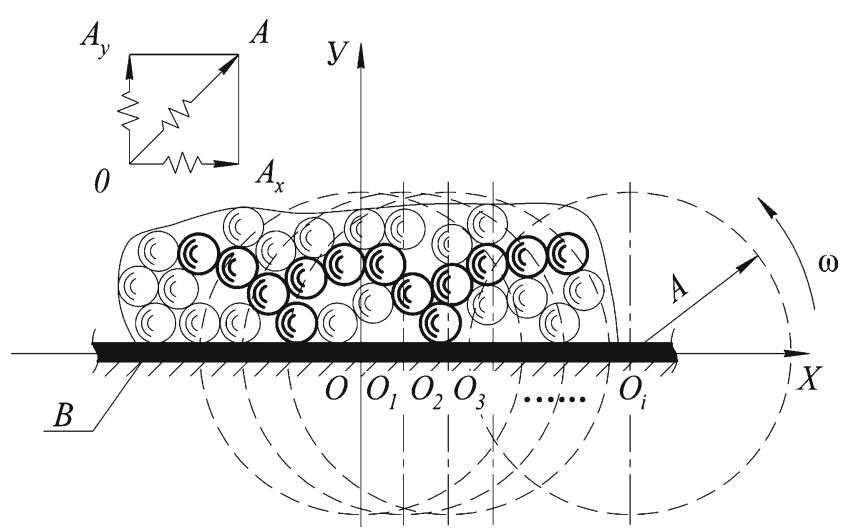

Fig. 2 Character of motion of the working medium granules at a planed oscillating surface 
The working medium granules fill all of overhead space $(y>0)$, above a surface $B$, any point of which moves according to the law:

$$
\begin{aligned}
& V_{0 y}=V_{0}(t)=A \omega \cos \omega t \\
& V_{0 x}=U_{0}(t)=-A \omega \sin \omega t .
\end{aligned}
$$

time, may be expressed by means of Navier-Stokes equations, the continuity equation, and the equation of state of gas, which a in change internal energy is taken into account [17]:

$$
\frac{d \bar{V}}{d t}=\bar{F}-\frac{1}{\rho} \operatorname{grad} P+\nu \Delta \bar{V}+\left(\frac{\zeta}{\rho}+\frac{\nu}{3}\right) \operatorname{grad} \operatorname{div} \bar{V}
$$

Determination of dependence of the velocity field of the elementary volume of the working medium of granules, as well as their densities and pressures on the coordinates and

$$
\frac{\partial \rho}{\partial t}+\operatorname{div}(\rho \bar{V})=0
$$

The following notations are taken in Eqs. (3-5): $\bar{V}-$ velocity of motion of elementary volume of the working medium granules; $\bar{F}$ - strength of the mass forces field; $\rho-$ density of the working medium; $\nu=\eta / \rho$ and $\eta-$ constant coefficients of kinematics and dynamic viscosity correspondingly; $P$ - pressure in the elementary volume of the working medium; $\zeta$ - constant coefficient of volume viscosity; $U$ internal energy per unit mass of the working medium; $K$ thermal conductivity; and $T$-temperature of elementary volume of working medium.

The presented Eqs. (3-5) can be complemented by the equation for the enthalpy of the working medium $H$ :

$$
\begin{aligned}
\rho \frac{d H}{d t}= & \operatorname{div}(K \operatorname{grad} T)+\left(\eta \left\{2\left[\left(\frac{\partial V_{x}}{\partial x}\right)^{2}+\left(\frac{\partial V_{y}}{\partial y}\right)^{2}+\left(\frac{\partial V_{z}}{\partial z}\right)^{2}\right]+\right.\right. \\
& \left.\left.+\left[\left(\frac{\partial V_{x}}{\partial y}+\frac{\partial V_{y}}{\partial x}\right)^{2}+\left(\frac{\partial V_{x}}{\partial z}+\frac{\partial V_{z}}{\partial x}\right)^{2}+\left(\frac{\partial V_{y}}{\partial z}+\frac{\partial V_{z}}{\partial y}\right)^{2}\right]\right\}+\left(\zeta-\frac{2}{3} \eta\right)(\operatorname{div} \bar{V})^{2}\right) .
\end{aligned}
$$

The above Eqs. (3-6) allow to determine six unknown quantities: $\bar{V}=\left(i V_{x}+j V_{y}+k V_{z}\right), \rho, P, T$. However, the search of solutions of these equations in a general view even for simple initial and boundary conditions satisfies insuperable mathematical difficulties practically $[18,19]$. In this connection, all of the found solutions have been obtained by the simplifications with taking into account the physically grounded assumptions.

In this case, one of such mentioned assumptions is the constancy of density of the working medium in its elementary volume. It is confirmed by the monitoring of motion of granules in the process of vibration treatment, when $\operatorname{div} \bar{V} \equiv 0$ [16]. The second assumption is the absence of convective acceleration [12] which is described as a term $(\bar{V} \Delta) \bar{V}$ and also named the Lagrange derivate, basic derivate [20], or derivate of Stoksa [21]. This is certainly executed for derivatives $\frac{\partial}{\partial x}$ and $\frac{\partial}{\partial z}$, as the flow pattern of convection does not change along axes $O X$ and $O Z$.

Let us estimate remaining convective terms $V_{y} \frac{\partial V_{x}}{\partial y}$ and $V_{y} \frac{\partial V_{y}}{\partial y}$ in comparison with the quantities of $\frac{\partial V_{x}}{\partial t}$ and $\frac{\partial V_{y}}{\partial t}$. It is known from the work [11] that the velocity of granules motion of the working medium diminishes with a distance from the wall of oscillating reservoir to its central part. This is due to the damping of power impulse caused by the damping and dissipative properties of the working medium. A maximal distance 
at which takes place the complete damping of power impulse makes no less than $200 \ldots 250 \mathrm{~mm}$. It is adjusted by the modes of motion of reservoir at the amplitude of its vibrations 1.0... $5.0 \mathrm{~mm}$ and at frequency $30 \ldots 70 \mathrm{~Hz}$, and also by the size of the working medium granules.

The expressions of maximal values of motion velocities $V_{x}$ and $V_{y}$ of the reservoir include the product $A \omega$, where $A$ and $\omega$ are the amplitude and frequency of vibrations of reservoir, respectively. Therefore, when doing the following estimations, then: $\frac{\partial V_{x}}{\partial t} \sim \frac{\partial V_{y}}{\partial t} \sim \omega V_{y} \sim A \omega^{2}$ and $V_{y} \frac{\partial V_{x}}{\partial y} \sim V_{y} \frac{\partial V_{y}}{\partial y} \sim A^{2} \omega^{2}$. At comparison of the estimations, we obtain expressions $\frac{\partial V_{x}}{\partial t} \gg$ $V_{y} \frac{\partial V_{x}}{\partial y}$ and $\frac{\partial V_{y}}{\partial t} \gg V_{y} \frac{\partial V_{y}}{\partial y}$, from which it follows that the convective terms can be neglected in the Eqs. (3-6).

After the conducted simplifications the Navier-Stokes Eq. (3) are accepted such as:

$$
\begin{aligned}
& \frac{\partial V_{x}}{\partial t}=F_{x}-\frac{1}{\rho} \frac{\partial P}{\partial x}+\nu\left(\frac{\partial^{2} V_{x}}{\partial y^{2}}+\frac{\partial^{2} V_{x}}{\partial x^{2}}+\frac{\partial^{2} V_{x}}{\partial z^{2}}\right) ; \\
& \frac{\partial V_{y}}{\partial t}=F_{y}-\frac{1}{\rho} \frac{\partial P}{\partial y}+\nu\left(\frac{\partial^{2} V_{y}}{\partial y^{2}}+\frac{\partial^{2} V_{y}}{\partial x^{2}}+\frac{\partial^{2} V_{y}}{\partial z^{2}}\right) .
\end{aligned}
$$

Since any changes in the convection flow pattern along the axes $O X$ and $O Z$ is not observed, then: $\frac{\partial P}{\partial x} \equiv 0$ and $\frac{\partial^{2} V_{x}}{\partial x^{2}}=$ $\frac{\partial^{2} V_{x}}{\partial z^{2}}=\frac{\partial^{2} V_{y}}{\partial x^{2}}=\frac{\partial^{2} V_{y}}{\partial z^{2}} \equiv 0$.

Thus, the final form of the Navier-Stokes Eqs. (7) and (8) for determination of the velocity components $V_{x}$ and $V_{y}$ of motion velocity $V$ of the working medium is as follows:

$$
\left\{\begin{array}{l}
\frac{\partial V_{x}}{\partial t}=F_{x}+\nu \frac{\partial^{2} V_{x}}{\partial y^{2}} \\
\frac{\partial V_{y}}{\partial t}=F_{y}-\frac{1}{\rho} \frac{\partial P}{\partial y}+\nu \frac{\partial^{2} V_{y}}{\partial y^{2}} .
\end{array}\right.
$$

Further, not losing community, we neglect the permanent terms $F_{x}$ and $F_{y}$ of equations system (9) which can be solved independently. In the second equation of the system (9) as an unknown term remains $\frac{\partial P}{\partial y}$.

The boundary conditions of motion velocity of by-wall flow of the working medium granules, which are at the planed wall of the reservoir, accomplishing oscillating motions on the circular trajectory, are the following:

$V_{0 x}=-A \omega \sin \omega t$

$V_{0 y}=A \omega \cos \omega t$.

Obviously, at the indicated boundary conditions (10) and (11), the changes in the movement of working medium flow will take a place only in relation to the axis $O Y$, namely, along the planed walls of the reservoir. The granules will start their movements from the state of its static equilibrium and then, during the insignificant interval of time from the beginning of motion of the reservoir, the velocity field of the working medium granules will be in transitional regime, depending on the conditions of stabilizing of the amplitude-frequency characteristics of the reservoir. At any time after stabilization, the motion velocity of granules will acquire a character of harmonic function, having temporal dependence. In the steady operating regime, the frequency of motion of the granules of by-wall flow is subject to the harmonic law and coincides with the frequency of the oscillating motion of the reservoir [22].

\section{Solution of equations of motion of the working medium at the planed oscillating surface}

To solve the equations, system (9) of motion of the working medium at the oscillating planed surface need not be examined, taking a place at the beginning of the cycle short-term transient regime from preresonance one, passing the phenomenon of resonance, to postresonance mode, at which the motion velocity of granules will acquire a character of harmonic function. It is explained that the process of finishinggrinding treatment of the parts is executed a long time in a few orders exceeding the time of transient mode. Thus, the solution of the first equation of the system (9) can be presented as:

$V_{x}(y, t)=e^{i \omega t} E(y)$.

Substituting expression (12) in the first equation of the system (9), we get:

$i \omega F=\nu \frac{\partial^{2} F}{\partial y^{2}}$.

The unique solution of Eq. (13), remaining finite over $y \rightarrow$ $\infty$, is the following:

$F(y)=\lambda e^{-(1+i) \sqrt{\omega / 2 v y}}$.

Substituting boundary values (10) for velocity $V_{x}$, we find the final solution of Eq. (12):

$V_{x}=-A \omega e^{-\sqrt{\omega / 2 v y}} \sin (\omega t-\sqrt{\omega / 2 v y})$.

Expression (15) for velocity $V_{x}$ can be considered as a damped wave, length of which is equal to $2 \pi \sqrt{\frac{2 \nu}{\omega}}$. The wave propagates in the direction of axis $O Y$ with phase velocity 
$\sqrt{2 \nu \omega}$ and falls slowly in the same direction according to the law $e^{-\sqrt{\omega / 2 v y}}$.

The system of Eq. (9) has rectilinear character, and both its equations are independent. In this context, to determine the velocity $V_{y}$ we consider the second equation of the system (9) separately. As well as in the case of finding the velocity $V_{x}$, mass force $F_{y}$ is neglected. Then the second equation of the system (9) will be presented in the following form:

$\frac{\partial V_{y}}{\partial t}=-\frac{1}{\rho} \frac{\partial P}{\partial y}+\nu \frac{\partial^{2} V_{y}}{\partial y^{2}}$.

To solve the Eq. (16), it is necessary to determine the general view of the function $P(y, t)$. Without taking into account the gravity $\left(F_{y}=0\right)$, pressure change along the axis $O Y$ will depend on the change of value of velocity $V_{y}$. Attenuation of the velocity $V_{y}$, depending on a quantity $y$, will have the same form as the damping the velocity $V_{x}$. This follows from the Eq. (16) which differs from the first equation of the system (9) only by the term $-\frac{1}{\rho} \frac{\partial P}{\partial y}$. Therefore, we determine dependence $P(y, t)$ as follows:

$P(y, t)=H(\omega) e^{i \omega t} e^{-2(1+i) \sqrt{\omega / 2 v y}}$.

The boundary conditions for the pressure quantity $P$ at $y=$ 0 must satisfy the condition at which $P(0, t)=C_{P} \rho \omega^{2} A^{2} e^{i \omega t}$, where $\widetilde{N}_{P} \approx \frac{1}{2}$, and term $e^{i \omega t}$ is determined, coming from the boundary conditions of motion of the planed surface.

Substituting the dependence (17) in Eq. (16), we get inhomogeneous equation of heat conduction:

$\frac{\partial V_{y}}{\partial t}=\nu \frac{\partial^{2} V_{y}}{\partial y^{2}}+C_{P} \omega^{2} A^{2} e^{i \omega t} 2(i+1) \sqrt{\omega / 2 v} e^{-2(1+i) \sqrt{\omega / 2 v} y}$.

The solution of the homogeneous part of Eq. (18) has the same form as the solution of Eq. (15) for the velocity $V_{x}$. Using this solution, we obtain:

$V_{y}^{*}=g(\omega) e^{i \omega t} e^{-(1+i) \sqrt{\omega / 2 v y}}$.

The dependence $g(\omega)$ in expression (19) is presented as a function of vibrations frequency $\omega$ of the planed wall of the reservoir, which will be determined in future.

The general solution of Eq. (16) is determined as the sum $V_{y}=V_{y}^{*}+\widetilde{V}_{y}$, where $\widetilde{V}_{y}$ is presented in the form:

$\bar{V}_{y}=f(t) e^{-2(1+i) \sqrt{\omega / 2 v y}}$.
Substituting the expression (20) in Eq. (16), we get the following relation for the dependence $f(t)$ :

$f^{\prime}=i 4 \omega f+2 C_{P} A^{2} \omega^{2} e^{i \omega t}(1+i) \sqrt{\frac{\omega}{2 \nu}}$.

Expression (21) is an inhomogeneous equation in ordinary derivatives. The solution of the homogeneous part of this equation looks like as follows:

$f=c e^{i 4 \omega t}$,

where $c$ is taken as a temporal function.

Further, using the standard procedure of method of indefinite coefficients [23] for finding the solution of the inhomogeneous Eq. (21), we get the following:

$f(t)=c(t) e^{i \omega t}$.

$f^{\prime}=c^{\prime} e^{i \omega t}+c i \omega e^{i \omega t}$.

Substituting got relationships (23) and (24) in Eq. (21), we get the expression for $c$ :

$c^{\prime}=2 C_{P} A^{2} \omega^{2}(1+i) \sqrt{\frac{\omega}{2 \nu}} e^{-i 3 \omega t}$.

$c=-\frac{2}{3} C_{P} A^{2} \omega \sqrt{\frac{\omega}{2 \nu}}(1-i) e^{-i 3 \omega t}+c_{0}$.

Further substitutions show that $c_{0}=0$, so the expression for the velocity $\bar{V}_{y}$ becomes:

$\widetilde{V}_{y}=-\frac{2}{3} C_{P} A^{2} \omega \sqrt{\frac{\omega}{2 \nu}}(1-i) e^{-i \omega t} e^{-2(1+i) \sqrt{\omega / 2 v y}}$.

Using the relationships (19) and (27), we get the general solution of Eq. (16):

$V_{y}=g(\omega) e^{i \omega t} e^{-(1+i) \sqrt{\omega / 2 v y}}-\frac{2}{3} C_{P} A^{2} \omega \sqrt{\frac{\omega}{2 \nu}}(1-i) e^{i \omega t} e^{-2(1+i) \sqrt{\omega / 2 v y}}$.

The expression (28) for the velocity $V_{y}$ at $y=0$ must fulfill the boundary condition which in a complex form is the following ratio:

$\left.V_{y}\right|_{y=0}=A \omega e^{i \omega t}$. 
Substituting the boundary condition (29) in general expression (28) for the velocity $V_{y}$, we find $g(\omega)$, that is:

$g(\omega)=A \omega\left(1+\frac{2}{3} C_{P} A \sqrt{\frac{\omega}{2 \nu}}(1-i)\right)$.

From the expression (30), we determine the final expression for the velocity $V_{y}$ in complex form, corresponding to the boundary condition (29):

$$
\begin{gathered}
V_{y}=A \omega e^{i \omega t}\left[\left(1+\frac{2}{3} C_{P} A \sqrt{\frac{\omega}{2 \nu}}(1-i)\right) e^{-(i+1) \sqrt{\omega / 2 v y}}-\right. \\
\left.-\frac{2}{3} C_{P} A \sqrt{\frac{\omega}{2 \nu}}(1-i) e^{-2(i+1) \sqrt{\omega / 2 v y}}\right] .
\end{gathered}
$$

The real part of the expression for the velocity $V_{y}$, which satisfies Eq. (16), and the boundary condition, $V_{y}(0, t)=$ $A \omega \cos \omega t$, looks like in the form:

$$
\begin{aligned}
\operatorname{Re}\left(V_{y}\right)= & A \omega\left[e ^ { - \sqrt { \omega / 2 v y } } \left(\left(1+\frac{2}{3} C_{P} A \sqrt{\frac{\omega}{2 \nu}}\right) \cos \left(\omega t-\sqrt{\frac{\omega}{2 \nu}} y\right)+\right.\right. \\
& \left.+\frac{2}{3} C_{P} A \sqrt{\frac{\omega}{2 \nu}} \sin \left(\omega t-\sqrt{\frac{\omega}{2 \nu}} y\right)\right)-\frac{2}{3} C_{P} A \sqrt{\frac{\omega}{2 \nu}} e^{-2 \sqrt{\omega / 2 v y}} \times \\
& \left.\times\left(\cos \left(\omega t-2 \sqrt{\frac{\omega}{2 \nu}} y\right)+\sin \left(\omega t-2 \sqrt{\frac{\omega}{2 \nu}} y\right)\right)\right]
\end{aligned}
$$

Expression (32) for the velocity $V_{y}$, as well as the expression for the velocity $V_{x}$, can be examined as a superposition of attenuated waves. However, unlike the expression for the velocity $V_{x}$ in the expression (32) for the vertical velocity component $V_{y}$ in multi-layered flow of the working medium granules there are two types of waves which correspond to two types of damping. So, along with waves, having length $2 \pi \sqrt{\frac{2 \nu}{\omega}}$, which are propagating with the velocity $\sqrt{2 \nu \omega}$ and are damping under the law $e^{-\sqrt{\omega / 2 v y}}$, there are the with length $2 \pi \sqrt{\frac{\nu}{2 \omega}}$, in the working medium. The waves are propagating along the axis $O Y$ with the velocity $\sqrt{\frac{\nu \omega}{2}}$ and are damping under the law $e^{-2 \sqrt{\omega / 2 v y}}$.

Thus, the solutions of the equations system (9) taking into account the boundary conditions, represented by the expressions $\left.V_{x}\right|_{y=0}=-A \omega \sin \omega t$ and $\left.V_{y}\right|_{y=0}=A \omega \cos \omega t$, are the followings relations:

$$
\left\{\begin{aligned}
V_{x}(y, t) & =-A \omega e^{-\sqrt{\omega / 2 v y}} \sin \left(\omega t-\sqrt{\frac{\omega}{2 \nu}} y\right) \\
V_{y}(y, t) & =A \omega\left[e ^ { - \sqrt { \omega / 2 v y } } \left(\left(1+\frac{2}{3} C_{P} A \sqrt{\frac{\omega}{2 \nu}}\right) \cos \left(\omega t-\sqrt{\frac{\omega}{2 \nu}} y\right)+\right.\right. \\
& \left.+\frac{2}{3} C_{P} A \sqrt{\frac{\omega}{2 \nu}} \sin \left(\omega t-\sqrt{\frac{\omega}{2 \nu}} y\right)\right)-\frac{2}{3} C_{P} A \sqrt{\frac{\omega}{2 \nu}} e^{-2 \sqrt{\omega / 2 v y}} \times \\
& \left.\times\left(\cos \left(\omega t-2 \sqrt{\frac{\omega}{2 \nu}} y\right)+\sin \left(\omega t-2 \sqrt{\frac{\omega}{2 \nu}} y\right)\right)\right]
\end{aligned}\right.
$$

Thus, the expression (17) for determination of pressure in the multi-layered flow of the working medium granules is as follows:

$P(y, t)=C_{P} \rho \omega^{2} A^{2} e^{-2 \sqrt{\omega / 2 v y}} \cos \left(\omega t-2 \sqrt{\frac{\omega}{2 \nu}} y\right)$.

The expression (34) is presented as a damped wave which is propagating with the phase velocity $\sqrt{\frac{\nu \omega}{2}}$ along the axis $O Y$, then it is damping in the same direction under the law $e^{-2 \sqrt{\omega / 2 v y}}$ and has a wavelength $2 \pi \sqrt{\frac{\nu}{2 \omega}}$.

It is necessary to add that the waves described higher are analogical not acoustic, but thermal waves [24, 25].

Quantity $\nu$, which characterizes the kinematic viscosity of multi-layered flow of the working medium granules, is determined by the attenuation of the motion velocity of the granules as a result of damping effect of the working medium. In addition, there are some methods to determine experimentally the coefficient $\sqrt{\frac{\omega}{2 \nu}}$, characterizing the damping of the motion velocity of the granules [26].

Examining the reasons of the effect of structural components of the damping coefficient of the motion velocity of the granules on the mechanism of vibration treatment process it should be added that in the theory of gas, the laws of which we follow, kinematics viscosity $\nu \sim V_{\mathrm{m}}$, where $V_{\mathrm{m}}$ is the average motion velocity of gas molecules. In our case, at motion of the by-wall multi-layered flow of granules of the working medium in the oscillating reservoir similarity $V_{\mathrm{gr}} \sim A \omega$ is executed. So, we can conclude that the coefficient $\sqrt{\frac{\omega}{2 \nu}}$ should not depend on the frequency of vibrations of the reservoir.

\section{Solution of equations of motion of the working medium for the conditions of the oscillating reservoir}

Expressions (33), being the solution of the system (9) at $y=0$, are transformed in the followings relationships:

$V_{x}=-A \omega \sin \omega t$,

$V_{y}=A \omega \cos \omega t$.

The set relationships (35) and (36) describe the kinematics of granules of by-wall flow of the working medium in the reservoir, where each point of planed working surface accomplishes oscillating motion along the trajectory close to a circle. Such kinematics results in forming of circulation and oscillation of the granules of multi-layered by-wall flow, the motion of which is directed oppositely to the motion of the reservoir walls. Forming of features of flow motion is explained that the granules do not repeat to a full degree the motion pattern of the planed working surfaces of the oscillating reservoir. It is 
associated with damping and dissipative properties of the working medium because of power losses.

Let us suppose that in the moment of time $t_{0}$, when the velocity $V_{y}$ of the wall takes a negative value, the separation of granules of by-wall flow takes place from the wall of the reservoir (Fig. 3). At this point the vertical components of motion velocity of the granules of by-wall flow become less than the vertical component of wall motion of the reservoir, i.e., $A \omega \cos \omega t \geq A \omega \cos \alpha_{0}$. Not taking into account the action of gravity, it can be noted that by-wall flow of the working medium granules will begin rectilinear motion with velocity $A \omega$ at an angle to the wall plane of the reservoir, i.e. $\alpha_{0}=\omega t_{0}$. This rectilinear motion will be maintained to the moment of time $t^{*}=\alpha^{*} / \omega$, when the reservoir wall during its circular moving again will enter into a contact with the working medium granules, accomplishing short-time independent motion.

Moment of time $t^{*}=\alpha^{*} / \omega$ can be determined from the equality of displacements in the vertical direction of the reservoir wall and the by-wall flow of granules. Vertical displacement of the wall $h_{\mathrm{rw}}$ for a time interval from $t_{0}$ to $t^{*}$ is determined by the following relation:

$$
\begin{aligned}
h_{r w} & =\int_{t_{0}}^{t^{*}} \mathrm{~V}_{y}(t) d t=\int_{t_{0}}^{t^{*}} \mathrm{~A} \omega \cos (\omega t)=A\left(\sin \omega t^{*}-\sin \omega t_{0}\right) \\
& =A\left(\sin \alpha^{*}-\sin \alpha_{0}\right) .
\end{aligned}
$$

The vertical displacement of granules $h_{\mathrm{gr}}$ in the by-wall flow for the same period of time, i.e., from $t_{0}$ to $t^{*}$, will be equal:

$h_{\mathrm{gr}}=A \omega\left(t^{*}-t_{0}\right) \cos \omega t_{0}=A\left(\alpha^{*}-\alpha_{0}\right) \cos \omega t_{0}$.

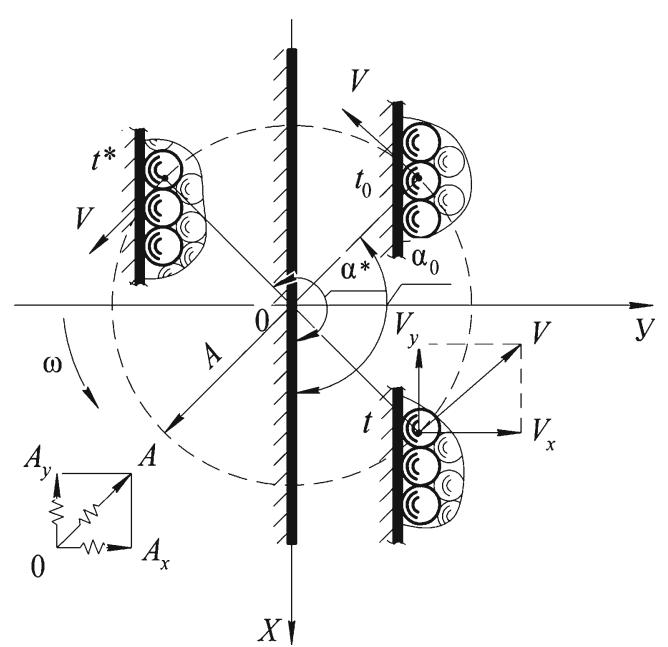

Fig. 3 Motion pattern of the working medium granules at the oscillating wall of the reservoir
Equating expressions (37) and (38), we get the following equation:

$\sin \alpha_{0}-\alpha_{0} \cos \alpha_{0}+\alpha^{*} \cos \alpha_{0}-\sin \alpha^{*}=0$.

Thus, the motion of the by-wall flow of working medium granules can be described as follows:

- longitudinally the axis $O Y$ :

$$
\begin{aligned}
& V_{1 y}=A \omega \cos \omega t \text { at } 0 \leq t<t_{0}=\alpha_{0} / \omega ; \\
& V_{2 y}=A \omega \cos \omega t_{0}=A \omega \cos \alpha_{0} \text { at } t_{0} \leq t<t^{*}, \text { i.e. } \frac{\alpha_{0}}{\omega} \leq t<\frac{\alpha^{*}}{\omega} ; \\
& V_{3 y}=A \omega \cos \omega t \text { at } t^{*} \leq t<T=\frac{2 \pi}{\omega}, \text { i.e. } \frac{\alpha^{*}}{\omega} \leq t<\frac{2 \pi}{\omega} ;
\end{aligned}
$$

- longitudinally the axis $O X$ :

$$
\begin{aligned}
& V_{1 x}=-A \omega \sin \omega t \text { at } 0 \leq t<t_{0}=\alpha_{0} / \omega ; \\
& V_{2 x}=-A \omega \sin \omega t_{0}=-A \omega \sin \alpha_{0} \text { at } t_{0} \leq t<t^{*}=\frac{\alpha^{*}}{\omega} \\
& V_{3 x}=-A \omega \sin \omega t \text { at } t^{*} \leq t<T=\frac{2 \pi}{\omega}
\end{aligned}
$$

Changing the motion velocity of the by-wall flow of granules in this case is illustrated by the graphic dependences (Fig. 4a, b). The proposed model of motion assumes that in the moment of time $t^{*}$ the motion velocity of the working medium granules becomes the equal to the motion velocity of the wall of the oscillating reservoir.

Described above the relationships (40) and (41) for the motion velocity of the working medium are newly accepted boundary conditions for the solution of the system (9) within the framework of the offered model of interaction of the working medium with the oscillating planed wall of the reservoir.

The displacement of the flow of the working medium granules in vertical direction for one period of vibrations $T$ of the reservoir will be determined by the relation:

$$
\begin{aligned}
& A \omega\left(t^{*}-t_{0}\right) \cos \omega t_{0}+A \omega \int_{t^{*}}^{T+t_{0}} \cos \omega t=\left[\left(t^{*}-t_{0}\right) \omega \cos \omega t_{0}+\right. \\
& \left.+\left(\sin \omega t_{0}-\sin \omega t^{*}\right)\right] A=A\left[\left(\alpha^{*}-\alpha_{0}\right) \omega \cos \alpha_{0}+\sin \alpha_{0}-\sin \alpha^{*}\right]
\end{aligned}
$$

The relation (42) taking into account Eq. (39) is equated with a zero, i.e., the displacement of the by-wall flow of granules in vertical direction for period $T$ of the oscillating motion of the reservoir is nonexistent practically. 

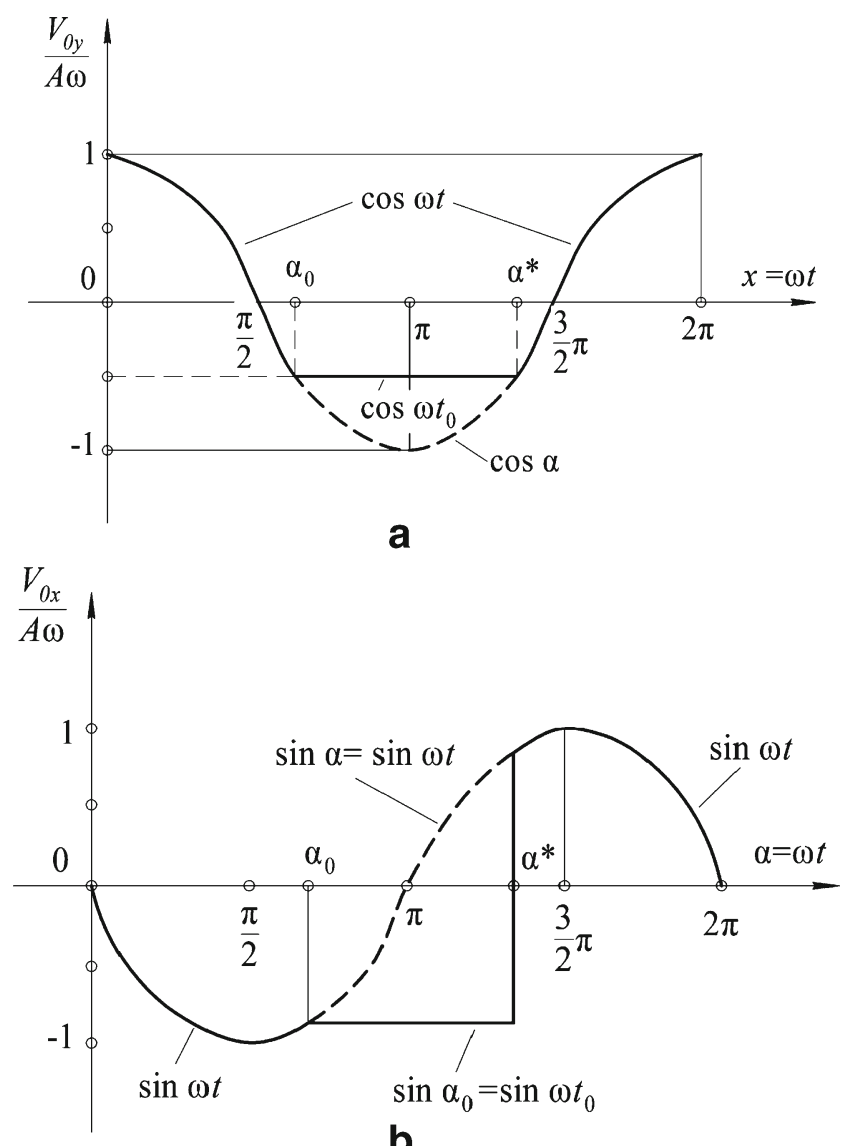

Fig. 4 Change of the motion velocity of granules of the by-wall flow of the working medium: a vertical motion velocity $V_{0 y} / A \omega$ and $\mathbf{b}$ horizontal motion velocity $V_{0 x} / A \omega$

The displacement of the flow of the working medium granules in horizontal direction is determined by the following equation:

$$
\begin{aligned}
& -A \omega\left[\left(t^{*}-t_{0}\right) \sin \alpha_{0}+\int_{\alpha^{*} / \omega}^{\left(T+t_{0}\right) / \omega} \sin \omega t\right] \\
& =-A\left[\left(\alpha^{*}-\alpha_{0}\right) \sin \alpha_{0}-\cos \alpha_{0}+\cos \alpha^{*}\right] .
\end{aligned}
$$

In Eq. (43), we accept that $T=\frac{2 \pi}{\omega}$. The quantity of the horizontal displacement of the flow of the working medium granules under the action of the reservoir wall is different from a zero and negative, that is the explanation of the opposition in the direction of the granules motion of the working medium flow and the wall of the oscillating reservoir.

If to expand the velocity of granules of the by-wall flow of the working medium into a Fourier series, then for the solution of the equations system (9) with new boundary conditions we can use the previously got solutions (32) and (34). In this case, the solution of the equations system (9) will be an endless sum of solutions with frequency $k \omega(k=1,2,3 \ldots \ldots)$, taken with certain coefficients, the values of which are determined at expansion of new boundary conditions into a Fourier series.

Let us designate the vertical and horizontal components of motion velocity of the working medium granules, accordingly through $V_{0}(t)=V_{0 y}(t)$ and $U_{0}(t)=V_{0 x}(t)$. The expansion into a Fourier series of both velocity constants looks like $[27,28]$ :

$$
\left\{\begin{array}{l}
U_{0}(t)=\frac{a_{0}^{U}}{2}+\sum_{k=1}^{\infty}\left(a_{k}^{U} \cos k \omega t+b_{k}^{U} \sin k \omega t\right) \\
V_{0}(t)=\frac{a_{0}^{V}}{2}+\sum_{k=1}^{\infty}\left(a_{k}^{V} \cos k \omega t+b_{k}^{V} \sin \omega t\right) .
\end{array} ;\right.
$$

On determination $a_{0}^{U}=\frac{2}{T} \int_{0}^{2 \pi / \omega} U_{0}(t) d t ; a_{0}^{V}=\frac{2}{T} \int_{0}^{2 \pi / \omega} V_{0}(t) d t$; $a_{k}^{U}=\frac{2}{T} \int_{0}^{2 \pi / \omega} U_{0}(t) \cos k \omega t d t ; \quad b_{k}^{U}=\frac{2}{T} \int_{0}^{2 \pi / \omega} U_{0}(t) \sin k \omega t d t$ $a_{k}^{V}=\frac{2}{T} \int_{0}^{2 \pi / \omega} V_{0}(t) \cos k \omega t d t ; \quad b_{k}^{V}=\frac{2}{T} \int_{0}^{2 \pi / \omega} V_{0}(t) \sin k \omega t d t$.

With newly accepted boundary conditions for $U(t)$ and $V(t)$, we have:

$$
\begin{aligned}
a_{0}^{U} & =-\frac{2 A \omega}{T}\left[\int_{0}^{\alpha_{0} / \omega} \sin \omega t d t+\sin \left(\omega t_{0}\right)\left(\frac{\alpha^{*}}{\omega}-\frac{\alpha_{0}}{\omega}\right)+\int_{\alpha^{*} / \omega}^{\alpha_{0} / \omega} \sin \omega t d t\right]= \\
& =-\frac{2 A}{T}\left[\left(\alpha^{*}-\alpha_{0}\right) \sin \alpha_{0}+\cos \alpha^{*}-\cos \alpha_{0}\right] .
\end{aligned}
$$

$$
\begin{aligned}
a_{0}^{V} & =-\frac{2 A \omega}{T}\left[\int_{0}^{\alpha_{0} / \omega} \cos \omega t+\cos \left(\omega t_{0}\right)\left(\frac{\alpha^{*}}{\omega}-\frac{\alpha_{0}}{\omega}\right)+\int_{\alpha^{*} / \omega}^{2 \pi / \omega} \cos \omega t d t\right]= \\
& =-\frac{2 A}{T}\left[\left(\sin \alpha_{0}-\sin \alpha^{*}\right)+\left(\alpha^{*}-\alpha_{0}\right) \cos \alpha_{0}\right] .
\end{aligned}
$$

From the Eq. (39) $a_{0}{ }^{V}=0$. We find other remaining coefficients in Fourier series of the system (44): 


$$
\begin{aligned}
& a_{k}^{U}=-\frac{2 A \omega}{T}\left[\int_{0}^{\alpha_{0} / \omega} \sin (\omega t) \cos (k \omega t) d t+\int_{\alpha_{0} / \omega}^{\alpha^{*} / \omega} \cos (k \omega t) \sin \left(\omega t_{0}\right) d t+\right. \\
& \left.+\int_{\alpha^{*} / \omega}^{2 \pi / \omega} \sin (\omega t) \cos (k \omega t) d t\right]=-\frac{2 A}{T}\left[\frac { 1 } { 2 ( k + 1 ) } \left(\cos \left(\alpha^{*}(k+1)\right)-\right.\right. \\
& \left.-\cos \left(\alpha_{0}(k+1)\right)\right)+\frac{1}{2(1-k)}\left(\cos \left(\alpha^{*}(1-k)\right)-\cos \left(\alpha_{0}(1-k)\right)\right)+ \\
& \left.+\frac{\sin \alpha_{0}}{k}\left(\sin \left(k \alpha^{*}\right)-\sin \left(k \alpha_{0}\right)\right)\right] \text {; } \\
& b_{k}^{U}=-\frac{2 A \omega}{T}\left[\int_{0}^{\alpha_{0} / \omega} \sin (\omega t) \sin (k \omega t) d t+\int_{\alpha_{0} / \omega}^{\alpha^{*} / \omega} \sin \alpha_{0} \sin (k \omega t) d t+\right. \\
& \left.+\int_{\alpha^{*} / \omega}^{2 \pi / \omega} \sin (\omega t) \sin (k \omega t) d t\right]=-\frac{2 A}{T}\left[\frac { 1 } { 2 ( k + 1 ) } \left(\sin \left(\alpha^{*}(k+1)\right)-\right.\right. \\
& \left.-\sin \left(\alpha_{0}(k+1)\right)\right)+\frac{1}{2(1-k)}\left(\sin \left(\alpha_{0}(1-k)\right)-\sin \left(\alpha^{*}(1-k)\right)\right)- \\
& \left.-\frac{\sin \alpha_{0}}{k}\left(\cos k \alpha^{*}-\cos k \alpha_{0}\right)\right] \text {; } \\
& a_{k}^{V}=\frac{2 A \omega}{T}\left[\int_{0}^{\alpha_{0} / \omega} \cos (\omega t) \cos (k \omega t) d t+\int_{\alpha_{0} / \omega}^{\alpha^{*} / \omega} \cos \alpha_{0} \cos (k \omega t) d t+\right. \\
& \left.+\int_{\alpha^{*} / \omega}^{2 \pi / \omega} \cos (\omega t) \cos (k \omega t) d t\right]=\frac{2 A}{T}\left[\frac { 1 } { 2 ( k + 1 ) } \left(\sin \left(\alpha_{0}(k+1)\right)-\right.\right. \\
& \left.-\sin \left(\alpha^{*}(k+1)\right)\right)+\frac{1}{2(1-k)}\left(\sin \left(\alpha_{0}(1-k)\right)-\sin \left(\alpha^{*}(1-k)\right)\right)+ \\
& \left.+\frac{\cos \alpha_{0}}{k}\left(\sin \left(k \alpha^{*}\right)-\sin \left(k \alpha_{0}\right)\right)\right] \\
& b_{k}^{V}=\frac{2 A \omega}{T}\left[\int_{0}^{\alpha_{0} / \omega} \cos (\omega t) \sin (k \omega t) d t+\int_{\alpha_{0} / \omega}^{\alpha^{*} / \omega} \cos \alpha_{0} \sin (k \omega t) d t+\right. \\
& \left.+\int^{2 \pi / \omega} \cos (\omega t) \sin (k \omega t) d t\right]=\frac{2 A}{T}\left[\frac { 1 } { 2 ( k + 1 ) } \left(\cos \left(\alpha^{*}(k+1)\right)-\right.\right. \\
& \alpha^{*} / \omega \\
& \left.-\cos \left(\alpha_{0}(k+1)\right)\right)+\frac{1}{2(1-k)}\left(\cos \left(\alpha_{0}(1-k)\right)-\cos \left(\alpha^{*}(1-k)\right)\right)+ \\
& \left.+\frac{\cos \alpha_{0}}{k}\left(\cos \alpha_{0}-\cos \alpha^{*}\right)\right]
\end{aligned}
$$

The expressions (47), (48), (49) and (50) are valid for the values $k=2,3,4 \ldots \infty$. But they have uncertainty at the values $k=1$.

The coefficients $a_{1}{ }^{U}$ and $b_{1}{ }^{V}$ can be determined from the general expressions, using the Lopital's rule. Then we get:

$$
\begin{aligned}
& a_{1}^{U}=-\frac{2 A}{T}\left[\frac{1}{4}\left(\cos 2 \alpha^{*}-\cos 2 \alpha_{0}\right)+\sin \alpha_{0}\left(\sin \alpha^{*}-\sin \alpha_{0}\right)\right] \\
& b_{1}^{V}=\frac{2 A}{T}\left[\frac{1}{4}\left(\cos 2 \alpha^{*}-\cos 2 \alpha_{0}\right)+\cos \alpha_{0}\left(\cos \alpha_{0}-\cos \alpha^{*}\right)\right] .
\end{aligned}
$$

For the coefficients $a_{1}{ }^{V}$ and $b_{1}{ }^{U}$ it is necessary to make integration of the expressions (48) and (49) at $k=1$. As a result, we get the following:

$$
\begin{aligned}
& b_{1}^{U}=-\frac{2 A \omega}{T}\left[\int_{0}^{\alpha_{0} / \omega} \sin ^{2}(\omega t) d t+\sin \alpha_{0} \int_{\alpha_{0} / \omega}^{\alpha^{*} / \omega} \sin (\omega t) d t+\right. \\
& \left.+\int_{\alpha^{*} / \omega}^{2 \pi / \omega} \sin ^{2}(\omega t) d t\right]=-\frac{2 A}{T}\left[\frac{1}{2}\left(\alpha_{0}-\alpha^{*}\right)+\pi+\frac{1}{4}\left(\sin 2 \alpha^{*}-\sin 2 \alpha_{0}\right)+\right. \\
& \left.+\sin \alpha_{0}\left(\cos \alpha^{*}-\cos \alpha_{0}\right)\right] . \\
& a_{1}^{V}=\frac{2 A \omega}{T}\left[\int_{0}^{\alpha_{0} / \omega} \cos ^{2}(\omega t) d t+\int_{\alpha_{0} / \omega}^{\alpha^{*} / \omega} \cos \alpha_{0} \cos (\omega t) d t+\right. \\
& \left.\quad+\int_{\alpha^{*} / \omega}^{2 \pi / \omega} \cos ^{2}(\omega t) d t\right]=\frac{2 A}{T}\left[\frac{1}{2}\left(\alpha_{0}-\alpha^{*}\right)+\pi+\frac{1}{4}\left(\sin 2 \alpha_{0}-\sin 2 \alpha^{*}\right)+\right. \\
& \left.+\cos \alpha_{0}\left(\sin \alpha^{*}-\sin \alpha_{0}\right)\right] .
\end{aligned}
$$

Thus, the final expressions for the velocities $U_{0}(t)$ and $V_{0}(t)$ on the surface of the reservoir-planed wall are as follows:

$$
\begin{aligned}
& U_{0}(t)=\frac{a_{0}^{U}}{2}+\sum_{k=1}^{\infty}\left(a_{k}^{U} \cos (k \omega t)+b_{k}^{U} \sin (k \omega t)\right) \\
& V_{0}(t)=\sum_{k=1}^{\infty}\left(a_{k}^{V} \cos (k \omega t)+b_{k}^{V} \sin (k \omega t)\right)
\end{aligned}
$$

Coefficient $\frac{\alpha_{0}^{U}}{2}$ determines the motion velocity of granules of the working medium by-wall flow, directed oppositely to motion of the oscillating reservoir wall. This coefficient depends on the quantities of the angles $\alpha_{0}$ and $\alpha^{*}$ of interaction of the reservoir wall with the working medium granules, i.e., on the quantity of the «separation» angle $\alpha_{0}$ and the angle $\alpha^{*}$ of subsequent «meeting» of the working medium granules with the container wall. The angles are determined from the expression (39), on the basis of which the graphic dependence has been got (Fig. 5).

It ensues from the graphic dependence (see Fig. 5) that at the «separation» angle $\alpha_{0}$ of the working medium granules from the reservoir wall, equal $\alpha_{0}=\pi / 2$, quantity of the angle $\alpha^{*}$ of their subsequent «meeting» is close to $2 \pi$. It means that the energy action of the reservoir planed walls on the working medium is virtually nonexistent during one period of its oscillation. With the growth of quantity of the angle $\alpha_{0}$ quantity of the angle $\alpha^{*}$ diminishes. It means that the time of action of the reservoir wall on the working medium is increased during one period of oscillation. Change of the motions velocity $V_{\mathrm{gr}}$ of granules of the working medium 
depending on the «separation» angle $\alpha_{0}$ of granules from the reservoir wall is shown by the graphic dependence (Fig. 6). The velocity $V_{\text {gr }}$ reaches its maximal value at $\alpha_{0}=\pi / 2$ and is equal $A \omega$.

Other members of the series in the expressions (55) and (56) are the boundary conditions for the solution of the equations system (9). The relations (33) and (34), taken with frequencies $\omega k$ and the coefficients $b_{k}{ }^{U}$ at $V_{x}(y, t)$ and $a_{k}{ }^{V}$ at $V_{y}(y, t)$, are the solutions of the equations system (9) for all of members of the series (55), containing functions $\sin (\omega k t)$ and for all of members of the series (56), containing the functions $\cos (\omega k t)$ :

$$
\begin{aligned}
V_{x k}(y, t) & =-b_{k}^{U} e^{-\sqrt{2 \omega k / v_{k} y}} \sin \left(\omega k t-\sqrt{\frac{2 \omega k}{\nu_{k}} y}\right) \\
V_{y k}(y, t) & =a_{k}^{V} e^{-\sqrt{\omega k / 2 v_{k} y}}\left(\left(1+\frac{2}{3} C_{P} \frac{a_{k}^{V}}{\omega k} \sqrt{\frac{\omega k}{2 \nu_{k}}}\right) \cos \left(\omega k t-\sqrt{\frac{\omega k}{2 \nu_{k}} y}\right)+\right. \\
& \left.+\frac{2}{3} C_{P} \frac{a_{k}^{V}}{\omega k} \sqrt{\frac{\omega k}{2 \nu_{k}}} \sin \left(\omega k t-\sqrt{\frac{\omega k}{2 \nu_{k}}} y\right)\right)-\frac{2}{3} C_{P} \frac{a_{k}^{V}}{\omega k} \sqrt{\frac{\omega k}{2 \nu_{k}}} \times \\
& \times e^{-2 \sqrt{\omega k / 2 v_{k} y}}\left(\cos \left(\omega k t-2 \sqrt{\frac{\omega k}{2 \nu_{k}}} y\right)+\sin \left(\omega k t-2 \sqrt{\frac{\omega k}{2 \nu_{k}}} y\right)\right) .
\end{aligned}
$$

For the terms of the series (55), containing the functions $\cos (\omega k t)$ and the terms of the series (56), containing functions $\sin (\omega k t)$ the corresponding solutions of the equations system (9) will be:

$$
\begin{aligned}
W_{x k}(y, t) & =-a_{k}^{U} e^{-\sqrt{\omega k / 2 v_{k} y}} \cos \left(\omega k t-\sqrt{\frac{\omega k}{2 \nu_{k}}} y\right) \\
W_{y k}(y, t) & =b_{k}^{V} e^{-\sqrt{\omega k / 2 v_{k} y}}\left(\left(1+\frac{2}{3} C_{P} \frac{b_{k}^{V}}{\omega k} \sqrt{\frac{\omega k}{2 \nu_{k}}}\right) \sin \left(\omega k t-\sqrt{\frac{\omega k}{2 \nu_{k}} y}\right)-\right. \\
& \left.-\frac{2}{3} C_{P} \frac{b_{k}^{V}}{\omega k} \sqrt{\frac{\omega k}{2 \nu_{k}}} \cos \left(\omega k t-\sqrt{\frac{\omega k}{2 \nu_{k}}} y\right)\right)+\frac{2}{3} C_{P} \frac{b_{k}^{V}}{\omega k} \sqrt{\frac{\omega k}{2 \nu_{k}}} \times \\
& \times e^{-2 \sqrt{\omega k / 2 v_{k} y}}\left(\cos \left(\omega k t-2 \sqrt{\frac{\omega k}{2 \nu_{k}}} y\right)-\sin \left(\omega k t-2 \sqrt{\frac{\omega k}{2 \nu_{k}}} y\right)\right) .
\end{aligned}
$$

The complete solution of the equations system (9) with the boundary conditions (55) and (56) is as follows:

$$
\left\{\begin{array}{l}
U_{\Sigma}=\frac{a_{0}}{2}+\sum_{k=1}^{\infty}\left(W_{x k}(y, t)+V_{x k}(y, t)\right) \\
V_{\Sigma}=\sum_{k=1}^{\infty}\left(V_{y k}(y, t)+W_{y k}(y, t)\right) .
\end{array}\right.
$$

In the system of Eq. (59), $U_{\Sigma}$ and $V_{\Sigma}$ are the horizontal and the vertical components of the motion velocity of the elementary volume of granules of the working medium by-wall flow.
In the expressions (57) and (58), the coefficients, determining the reduction of the motion velocity of the working medium granules along the axis $O Y$, have the form $\sqrt{\frac{\omega k}{2 \nu_{k}}}$ and 2 $\sqrt{\frac{\omega k}{2 \nu_{k}}}$; their quantities practically do not depend on the angular rotation velocity of the source of vibrations.

The dependence of pressure $P$ on the quantities $y$ and $t$, corresponding to the solution of the equations system (59) and boundary conditions (44), is as follows:

$$
\begin{aligned}
P_{\Sigma} & =\sum_{k=1}^{\infty} C_{P} \rho\left(\left(b_{k}^{V}\right)^{2} e^{-2 \sqrt{\omega k / 2 v_{k} y}} \sin \left(\omega k t-2 \sqrt{\frac{\omega k}{2 \nu_{k}}} y\right)+\right. \\
& \left.+\left(a_{k}^{V}\right)^{2} e^{-2 \sqrt{\omega k / 2 v_{k} y}} \cos \left(\omega k t-2 \sqrt{\frac{\omega k}{2 \nu_{k}} y}\right)\right) .
\end{aligned}
$$

\section{Numerical solutions of the motions velocity of the working medium in the oscillating reservoir}

The expressions (59) and (60) show that the horizontal $V_{x}$ and the vertical $V_{y}$ components of the motion velocity of granules, as well as the quantity of their pressure in the working medium flow depend not only on time of action of the oscillating reservoir wall to the working medium, but also on the coordinate $Y$, which indirectly characterizes the direction of granules motion. Consequently, the trajectory of motion of the working medium can be determined numerically in the form of temporal dependences of the horizontal and vertical components of the motion velocity of granules for four values of their distances from the planed wall of the oscillating reservoir (Fig. 7). The calculations have been carried out for the most used values of basic technological parameters of vibration treatment process, such as frequency and amplitude of

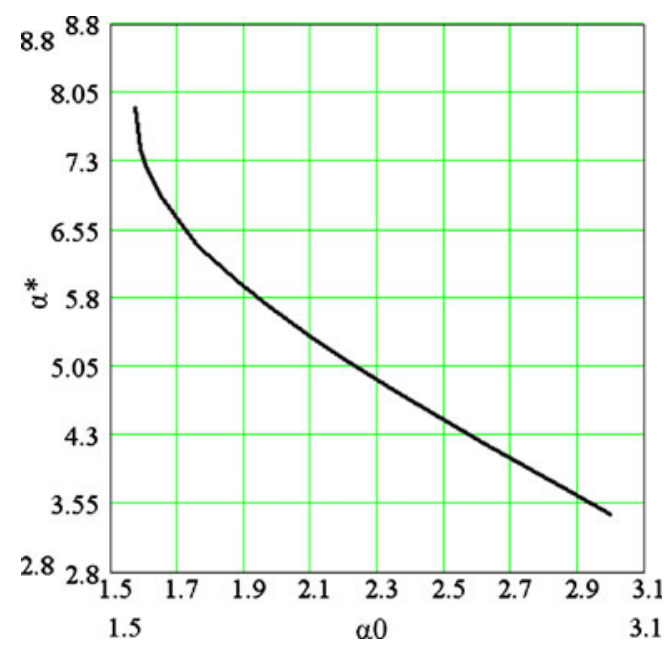

Fig. 5 Dependence of quantities of the «separation» angle $\alpha_{0}$ and the «meeting» angle $\alpha^{*}$ at interaction of the reservoir wall with the working medium granules 


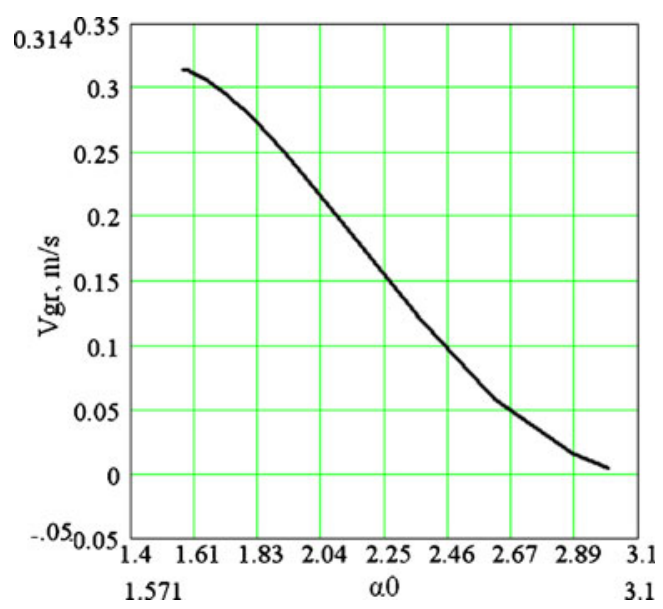

Fig. 6 Dependence of motions velocity $V_{\text {gr }}$ of granules of the working medium by-wall flow on the «separation» angle $\alpha_{0}$ of granules from the reservoir wall

vibrations $50 \mathrm{~Hz}$ and $1.0 \mathrm{~mm}[29,30]$, correspondingly; the angles of «separation» and «meeting» of the reservoir wall and the working medium granules 2,618 and 4,452 rad, correspondingly, with taking into account the distance from the reservoir wall to its central part, where the distance of complete damping of the force impulse makes $\approx 250 \mathrm{~mm}$ because of the inelastic collisions of granules [11].

The graphics of granules displacements of the working medium by-wall flow testify that the displacement along the axis $Y$ is a harmonic pattern (see Fig. 7). When the distance from the reservoir wall increases, the displacement amplitude falls. Also the phase of the oscillations is changed. The displacement along the axis $X$ is a superposition of harmonic vibrations and permanent displacement. The amplitude of vibrations along the axis $X$ also decreases while the distance from the wall of the reservoir to its central part increases.

The total displacement of granules of the working medium by-wall flow consists of vector sum of components of the motion velocity of granules (Fig. 8). The chart shows some variants of the motion trajectory of the working medium granules during five periods of vibrations of reservoir, thus

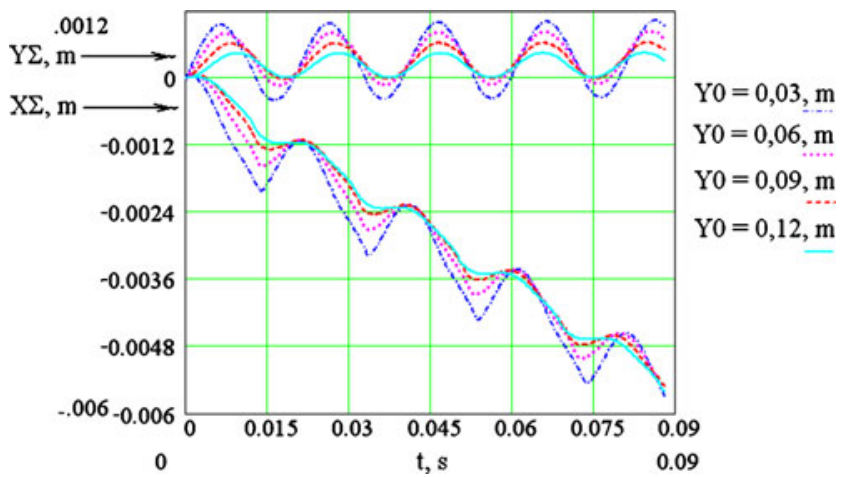

Fig. 7 Temporal dependence of quantities of the horizontal $\mathrm{X} \Sigma$ and vertical $Y \Sigma$ components of granules displacements of the working medium by-wall flow for different distances $Y_{0}$ from the reservoirs wall

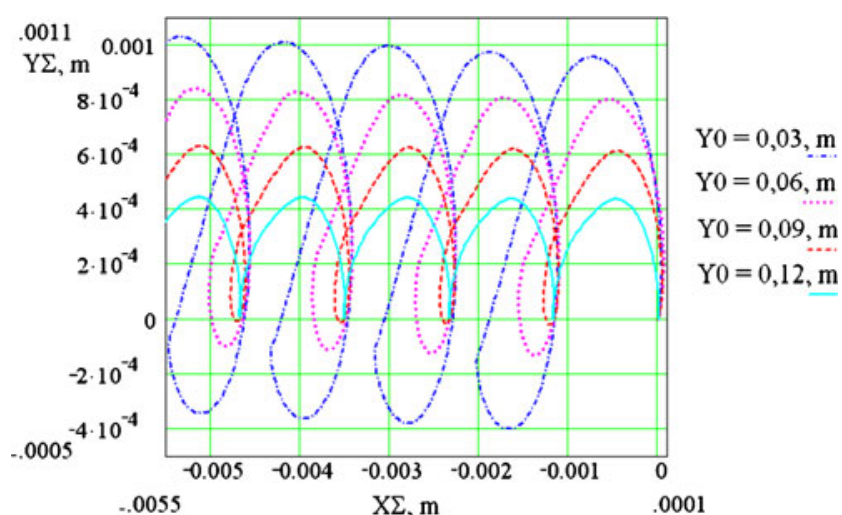

Fig. 8 Trajectory of the granules motion of the working medium by-wall flow during five periods of vibrations of the reservoir for different distances $Y_{0}$ from the wall of the reservoir

for each of variants of the trajectory the motion of granules begins at a certain distance $Y_{0}$ from the reservoir wall.

In addition, the graphs show that the working medium granules execute continuous motion on complex periodically recurring curves which are close to the particular type of cycloid-trochoid (see Fig. 8). When the distance from the wall of the reservoir to its central part increases, the amplitude of cycloids is reduced, and the looped character which is observed in the bottom of each trochoid disappears gradually. It is graphically shown also that in their motion the granules of multi-layered flows of the working medium are displaced from peripheral part near the walls of reservoir, where the displacement has a maximal value, to central part into depth of the reservoir. Thus the quantity of displacement during motion to central part is reduced because of the effect of damping and dissipation of the working medium. The initial value $Y_{0}$ of the distance of granules from the wall of reservoir corresponds to each of four curves.

The processes of micro-cutting and elastic-plastic deformation, which provide the mechanism of finishing-grinding treatment in the oscillating reservoir, take place at the relative moving and mutual pressure of the working medium granules and parts. In this connection, along with the estimation of kinematics of the working medium granules, it is expediently



Fig. 9 Dependence of pressure of granules in the multi-layered flow of the working medium on the quantity of distance of granules from the reservoir's wall 
to take into account the character of dependence of pressure in the multi-layered flow of the working medium on the displacement of the granules from the periphery to its central part.

The initial phase of pressure changes in the flow of working medium, concordant with a curve (Fig. 9), is selected so that $P(y)_{y=0}=0$. It should be marked that as far as the granules are distancing of from peripheral to central part of the reservoir, pressure can change a sign. Then the zone of rarefaction appears in the reservoir with the minimum quantities of the relative displacements and mutual pressure of the working medium granules and parts, that leads to decrease of activity of processing. At the distance $Y_{0} \approx 250 \mathrm{~mm}$, the pressure in the multi-layered flow of the working medium is reduced practically to the zero.

\section{Conclusions}

Mathematical modeling of motion of the working medium at finishing-grinding treatment in the oscillating reservoir of vibration machine, based on an analogy between the moving working medium granules and gas molecules allows to give the scientific explanation of the following physical effects, which are incident to the behavior of free working medium, executing the function of cutting tool in the processes of micro-cutting and elastic-plastic deformation, providing the achievement of the required technological result of finishinggrinding treatment.

Some of the effects, which are visually examined by the authors of the article and received a further mathematical ground, are the following:

- formation of the multi-layered by-wall flow of the working medium granules at the planed wall of reservoir, thus the direction of motion of the flow takes place oppositely to direction of the reservoir motion and source of its vibrations

- formation of motion of the working medium granules on trajectories in the form close to trochoids-cycloids

- formation of motion of granules of the multi-layered flows of the working medium at which the granules accomplish a drift from peripheral part of the walls of reservoir, where their displacement has a maximal value, to central part into depth of the reservoir, while during moving the displacement decreases because of the effect of damping and dissipation of the working medium

The above theoretical results are of fundamental character, and at the same time, the obtained mathematical conformities to the law specify that the offered method of simulation of motion of the working medium in the oscillating reservoir will give practical results, consisting of determination of such parameters of technology as amplitude, frequency and trajectory of reservoir's motion, as well as volume of its loading and correlation of working medium and processed parts in loading. The values of the indicated parameters quantitatively and qualitatively, by means of values of removing metal and obtained roughness of surface influence on achievement of concrete technological results of finishing-grinding vibration treatment. Among the results is possible to select the following:

- cleaning of castings and forgings from moulding and sticking sand

- cleaning from scale and different types of impurity

- rough polishing with the simultaneous removing the burrs and rounding off the sharp edges on the parts after machine-tool and forging-stamping operations

- clean polishing and polishing with diminishing of roughness to prepare the surface for electroplating and other kinds of surface impregnation

- Except for practical results, related to designing of new technologies of vibration treatment, and increase of the productivity and quality already existing technologies, the above mathematical conformities to the law can be used for the dynamic calculations of vibration exciter and other oscillating units of vibration machines, applied both on the operations of classic vibration processing and on the operations of its varieties. Charts of such operations can be obtained by combining the scheme of power action on the working medium and the processed parts, mounted in equipment, possessing by different kinematic properties and degrees of freedom.

\section{References}

1. Mitsyk A, Fedorovich V (2012) Classification of the methods of finishing-grinding treatment. The 3rd international scientifictechnical conference «Theory and practice of rational designing, producing and exploitation of engineering structures». November 7-9. Lvov. pp. 163-164

2. Mitsyk AV, Fedorovich VA (2012) Development of new technologies of vibration finishing-grinding and hardening treatment of the products of common-engineering application // Visnyk NTU «KhPI». Series New solutions in modern technologies. - Kharkov NTU «KhPI». - - No 47 (953). pp. 226-233

3. Kamyar H, Amirhossein M, Spelt JK (2013) Development of a laser displacement probe to measure particle impact velocities in vibrationally fluidized granular flows. Powder Technol 235:940-952

4. Mitsyk AV, Fedorovich VA, Fadeev VA (2012) The effectiveness of new kinds of vibration treatment method of products. Modern engineering technologies: printed scientific works. Kharkov NTU «KhPI» 7:74-81

5. Naeini SE, Spelt JK (2011) Development of single-cell bulk circulation in granular media in a vibrating bed. Powder Technol 211(1): 176-186

6. Sokołowska R, Majewski T (2007) Analysis of viscous-elastic model in vibratory processing. Recent Advances in Mechatronics. pp. 490-494 
7. Hashimoto F, DeBra DB (1996) Modelling and optimization of vibratory finishing process. CIRP Ann Manuf Technol 45(1):303306

8. Ciampini D, Papini M, Spelt JK (2008) Characterization of vibratory finishing using the Almen system. Wear 264(7-8):671-678

9. Ciampini D, Papini M, Spelt JK (2007) Impact velocity measurement of media in a vibratory finisher. J Mater Proc Technol 183(2-3):347-357

10. Babichev AP, Babichev IA (2008) The principles of vibration technology. Publishing centre of DSTU, Rostov-on-Don, p 694

11. Kartashov IN, Shainsky MYE, Vlasov VA (1975) The treatment of parts by free abrasives in vibrating reservoirs. Vyscha shkola, Kiev, p 188

12. Batchelor GK (2000) An introduction to fluid dynamics. Cambridge University Press, Cambridge, England, p 615

13. Patterson GN (1956) Molecular flow of gases. Wiley, New York, p 217

14. Hirschfelder JO, Charles F, Curtiss R, Bird B (1954) Molecular theory of gases and liquids. Wiley, New York, p 1219

15. Dudley RM (1999) Uniform central limit theorems. Cambridge University Press, Cambridge, England, p 436

16. Mitsyk AV Increase of the effectiveness of treatment of large-sized planed products by an activation of motion of the working medium in vibrating «U» - shaped containers. Thesis of candidate of technical sciences, 05.03.01. The dissertation is protected 10.04.2008, Kharkov NTU «KhPI», p 331

17. Yavorsky BM, Detlaf AA, Lebedev AK (2006) Reference book on physics, the 8th edn ONIKS. World and education, Moscow, p 1054

18. Peyret R, Taylor TD (1983) Computational methods for fluid flow. Springer, New York, p 358

19. Wesseling P (2001) Principles of computational fluid dynamics. Springer, Berlin, p 644
20. Tritton DJ (1989) Physical fluid dynamics, 2nd edn. Oxford University Press, Oxford, England, p 544

21. Kaplan W (1991) Advanced calculus, 4th edn. Addison-Wesley, Reading, MA, p 746

22. Goncharevich IF, Frolov KV (1981) The theory of vibration technics and technology. Science, Moscow, p 320

23. Riley KF, Hobson MP, Bence SJ (2010) Mathematical methods for physics and engineering. Cambridge University Press, Cambridge, England, p 1362

24. Kreith F, Black WZ (1980) Basic heat. Transfer Harper \& Row, New York, $\mathrm{p} 512$

25. Li Voti R, Liakhou GL, Paoloni S, Sibilia C, Bertolotti M (2001) Thermal waves physics. J Optoelectron Adv Mater 3(4):779816

26. Mitsyk AV, Fedorovich VA, Fadeev VA (2012) Development of the problems of cinematics and dynamics of finishing-grinding treatment in vibrating reservoir. Cutting $\&$ tool in technological system: International Scientific-Technical Collection. Kharkov NTU «KhPI» 82: $171-182$

27. Korner TW (1989) Fourier analysis. Cambridge University Press, Cambridge, England, p 591

28. Jordan DW, Smith P (1994) Mathematical techniques. Oxford University Press, Oxford, England, p 806

29. Babichev AP, Motrenko PD, Gillespie LK (2010) Employing vibrational technology for processing burr work on machined parts. Publishing centre of DSTU, Rostov-on-Don, p 289

30. Babichev AP, Motrenko PD, Lebedev VA (2006) The application of vibration technologies to increase the quality of surface and service properties of products. Publishing centre of DSTU, Rostov-on-Don, p 213 